\title{
Los estudios sobre la protesta social: el caso argentino
}

\section{The studies about the social protest: the Argentinian case}

\section{Diego Fernando Palacio Hoyos}

Universidad Nacional de Colombia

dfpalacioh@unal.edu.co

\section{Resumen}

En Argentina, la noción de "protesta social" se erigió como una alternativa a la noción de "movimientos sociales". El artículo aborda la protesta social a partir del análisis de los trabajos del Grupo de Estudios sobre Protesta Social y Acción Colectiva. La noción es influenciada por las investigaciones de la sociología política norteamericana y la ontología política de Laclau. La protesta social se constituiría en una muestra de la contingencia y de la posibilidad de una acción sin fundamento; para terminar como la unidad básica para abordar la acción colectiva. Para dar cuenta de estas características, el grupo propone un marco de análisis con cinco variables y, a partir de este marco, pretende dilucidar el vínculo entre las protestas sociales y las transformaciones sociopolíticas que se presentaron a partir de 1989 en Argentina. Sin embargo, el trabajo del grupo se enfrenta a una tensión entre su marco de análisis y su aplicación concreta.

Palabras clave: protesta social, movimientos sociales, acción colectiva, Argentina.

\begin{abstract}
In Argentina, the notion of "social protest" is presented as an alternative to the notion of "social movements". The article addresses the first based on the work of the Grupo de Estudios sobre Protesta Social y Accion Colectiva. The notion is influenced by the North American political sociology's investigations and Laclau's political ontology. The social protest is established as an example of the contingency and the possibility of an action without foundation; the notion is presented as the basic unit to approach the collective action. The group proposes an analysis framework with five variables and, with it, they pretend to elucidate the link between the social protests and the socio-political transformations that occurred since 1989 in Argentina. Nevertheless, the work of the group faces a tension between their analysis framework and its concrete application.
\end{abstract}

Keywords: social protest, social movements, collective action, Argentina.

Articulo: Recibido el 16 de septiembre de 2018 y aprobado el 29 de julio de 2019.

\section{Cómo citar este artículo:}

Palacios Hoyos, DF. (2019). Los estudios sobre la protesta social: el caso argentino. Reflexión Política 21 (42), pp. 145161. doi: $10.29375 / 01240781.3400$

\section{Introducción}

Los primeros años del siglo XXI llegaron con grandes movilizaciones sociales en América Latina que trajeron consigo cambios sociales y políticos en varios países de la región. Estos procesos sociales exigieron el despliegue de diversas herramientas teóricas que buscaban interpretar y explicar las transformaciones de 
la realidad social latinoamericana y sus actores. Así pues, en la región apareció una creciente bibliografía que buscaba dar cuenta de los movimientos sociales que se consideraron como protagonistas de las varias movilizaciones sociales. Las investigaciones en América Latina sobre los movimientos sociales reconfiguraron las concepciones clásicas y dominantes e, inclusive, se aventuraron en la construcción de teorías propias (Gohn, 2018).

Con todo, a pesar de las múltiples investigaciones, desde el Grupo de Investigación en Teoría Política Contemporánea - TEOPOCO-, luego de una aproximación preliminar, se identificó un gran desconocimiento de las formas como se han comprendido los movimientos sociales y la constitución de sujetos colectivos en América Latina. Para ayudar a subsanar dicho desconocimiento, se planteó un proyecto investigativo colectivo titulado Movimientos sociales y subjetividades: desafios teóricos desde América Latina ${ }^{1}$. Este proyecto planteó la siguiente pregunta básica: ¿cómo se construyen las teorías latinoamericanas referidas a la comprensión y/o explicación de los movimientos sociales y la constitución de sujetos colectivos entre 2000 y 2015 ?

Entre las múltiples opciones investigativas que brinda el periodo mencionado anteriormente, el contexto argentino es uno de los casos paradigmáticos en América Latina para el estudio de la movilización popular y los cambios sociales. Especialmente, desde el nacimiento del abigarrado movimiento peronista y la dictadura militar; las luchas centradas en los derechos humanos, tras la caída de la dictadura en la década de 1980; pasando por las luchas de los desocupados en la década de 1990 o los hechos ocurridos a partir de 2001, después de la crisis económica.

En la actualidad, en Argentina se pueden distinguir dos tendencias en los estudios de la acción colectiva: en un lado, se encuentran aquellos que realizan sus análisis a partir de la noción de "protesta social" y, en el otro, se encuentran aquellos que parten de una conceptualización específica a partir de la noción de "movimientos sociales" (Schuster, 2005; Schuster y Pereyra, 2001; Svampa, 2018). La primera noción se encuentra en los trabajos investigativos de Federico Schuster y su Grupo de Estudios sobre Protesta Social y Acción Colectiva (GEPSAC) o en los de Javier Auyero. Mientras que la segunda se encuentra, por ejemplo, en los trabajos de Maristella Svampa o Norma Giarracca.

Las dos nociones pueden rastrearse hasta dos diferentes influencias: la noción de "movimientos sociales" a partir de los trabajos de la sociología europea, representada por el paradigma de la identidad 2 , y la noción de "protesta social" desarrollada a partir de las investigaciones de la sociología política norteamericana y la llamada agenda clásica (Delgado, 2007) ${ }^{3}$.

Sin llegar a ahondar en las causas políticas, sociales y hasta económicas, se puede afirmar que en Argentina se tiene, como principal referente, los desarrollos teóricos y técnicas norteamericanas (Leiras, Medina y D’Alessandro, 2005, p. 81) ${ }^{4}$. Particularmente, en los estudios sobre los movimientos sociales y la acción colectiva, los enfoques basados en la agenda clásica son los dominantes actualmente (Svampa, 2009, p. 1).

Dentro del panorama anterior, este escrito pretende dar cuenta del enfoque que parte de la noción de "protesta social", dadas su centralidad e influencia. Características reflejadas en su constitución como lugar común, tanto en los medios de comunicación como en las esferas estatales y oficiales argentinas (Schuster et al., 2006, p. 18; Svampa, 2009, p. 4). Así pues, el presente escrito se centra en los estudios de la protesta social en Argentina; específicamente, los trabajos llevados a cabo por Federico Schuster y el GEPSAC, adscritos al Instituto de Investigaciones Gino Germani de la Facultad de Ciencias Sociales de la Universidad de Buenos Aires.

1 TEOPOCO es un grupo de la Universidad Nacional de Colombia, sede Bogotá, adscrito a la Facultad de Derecho, Ciencias Políticas y Sociales. El proyecto de investigación fue financiado por el Instituto Unidad de Investigaciones Jurídico Sociales Gerardo Molina _UNIJUS - de la misma Facultad.

2 Autores como A. Melucci, A. Touraine, M. Castells, C. Offe, A. Pizzorno, B. Klandermans y D. Della Porta, entre otros. 3 Con autores como M. Olson, Ch. Tilly, S. Tarrow, D. McAdam, J. McCarthy, A. Oberschall y M. Zald.

$4 \quad$ Leiras, Medina y D'Alessandro incluyen las aproximaciones de Schuster a la protesta social como parte de los desarrollos de la ciencia política argentina, al igual que Norma Giarracca (2001, p. 14). En el campo de la sociología, Javier Auyero ha desarrollado su carrera académica en Estados Unidos. 
El trabajo del GEPSAC se ha desarrollado a partir del proyecto titulado La acción colectiva y los procesos politicos de la construcción de la ciudadanía en la Argentina democrática. La transformación de la protesta social. La investigación se desarrolla, desde el año $1998^{5}$, alrededor del análisis de las transformaciones de la protesta social en Argentina a partir de $1989^{6}$. Inicialmente, el estudio abarcaba el periodo de 1989-1998; posteriormente, fue ampliado hasta 1999 (Schuster y Pereyra, 2001); luego hasta el 2003 (Schuster et al., 2006), para terminar, en la última iteración, hasta el 2007 (Pereyra, Pérez y Schuster, 2017).

Como parte del proyecto de investigación grupal de TEOPOCO, este escrito se pregunta por el cómo se produce teoría sobre los movimientos sociales; específicamente, por el cómo se produce la noción de protesta social en los trabajos de Schuster y el GEPSAC. Por consiguiente, su sentido no se encuentra en la producción de un conocimiento sobre la realidad social en forma directa, sino sobre la forma como se conoce esa realidad. En otras palabras, esta investigación no tiene como objeto principal los movimientos sociales o la protesta social en Argentina, en cuanto dimensiones empíricas susceptibles de ser estudiadas; antes bien, su enfoque se limita al estudio de la noción de protesta social en cuanto teoría utilizada para conocer determinada dimensión empírica.

Con la investigación se busca, en primer lugar, examinar críticamente los criterios, lógicas y metodologías en virtud de las cuales se producen y articulan los enunciados y conceptos que conforman la noción de protesta social, en calidad de teoría sobre los movimientos sociales y la acción colectiva; y, en segundo lugar, se busca comparar la noción de protesta social con las teorías dominantes sobre la acción colectiva y los movimientos sociales, en torno a sus fundamentos ontológicos y epistemológicos.
Para abordar el uso de la noción de protesta social a partir de los dos objetivos anteriores, el presente escrito se divide en cuatro partes. En la primera parte se muestran las generalidades del debate entre la noción de movimientos sociales y la noción de protesta social, con el fin de contextualizar el surgimiento de esta última. En la segunda parte, se presentan los presupuestos ontológicos y epistemológicos de los que parten los estudios basados en la protesta social en Argentina. En este apartado se busca mostrar cuál es la concepción de la realidad social de la que se parte - su ontología - y cómo plantea que debe ser la aproximación para conocer tal realidad - su epistemología-; al mismo tiempo, se lleva a cabo una comparación con las teorías dominantes sobre la acción colectiva y los movimientos sociales. La tercera parte se concentra en el marco de análisis que se pretende utilizar para abordar las protestas sociales. La última parte presenta la forma como, de hecho, se utiliza el marco de análisis propuesto en la actividad investigativa del GEPSAC.

\section{El debate: de los movimientos sociales a la protesta social}

En el contexto argentino, autores como Svampa (2009) y Schuster $(2005$; 2001) coinciden en que los estudios de la acción colectiva entre las décadas de 1970 y 1980 parecieron ir de la mano con los desarrollos del paradigma de la identidad, tras la crisis de las explicaciones a partir de las conceptualizaciones relacionadas con la "clase" o el "movimiento obrero"7. A partir de las teorías sobre los "nuevos movimientos sociales" se pretendió explicar y comprender los reclamos que fueron emergiendo con el fin de la dictadura y el proceso de transición democrática.

Más adelante, en la década de 1990, en el pasaje a un nuevo tipo societal basado en

5 Existe un antecedente del año anterior en la colaboración realizada por Schuster en el Informe anual sobre la situación de los Derechos Humanos en la Argentina 1996 (Centro de Estudios Legales y Sociales, 1997).

$6 \quad$ La decisión de escoger el año de 1989 como el año inicial del estudio obedeció a los procesos de reforma al Estado que se llevaron a cabo a partir de la llegada a la presidencia de Carlos Menem, en julio de 1989. Para los integrantes del GEPSAC, las reformas fueron un punto de quiebre en las formas y modalidades de protesta en Argentina.

Respecto a las reformas, Blutman (1994) identifica, en el periodo que va de 1989 hasta 1992, más de ochenta leyes y ochocientos decretos que tenían como objetivo cambiar la administración pública en Argentina. Esta producción normativa se reflejó en cambios de fondo en la estructura del Estado y la sociedad argentina: hubo cambios en el papel estatal —privatizaciones, desregulación, desmonopolización, etc.-, en la política económica — política fiscal, reducción del gasto público, la Ley de Convertibilidad, etc.—- organizacionales y reestructuración del aparato estatal y política de recursos humanos —reducción de funcionarios, profesionalización, relaciones sindicales, etc.-

$7 \quad$ Este paradigma de la identidad se desarrolló, en primera instancia, en Europa occidental, como respuesta a las limitaciones del modelo marxista para explicar las acciones colectivas ocurridas en los años sesenta. 
la globalización y el neoliberalismo, se vieron afectadas las acciones de los movimientos sociales y sus repertorios de movilización tradicionales. Como consecuencia de este escenario de fragmentación, diversificación y desarticulación de las luchas sociales, fueron puestos en entredicho los enfoques analíticos usados hasta ese momento y se le abrió el camino a nuevos conceptos y herramientas teóricas. A partir de las perspectivas de la agenda clásica se desarrolla la noción de "protesta social" como una alternativa ante la crisis de las identidades colectivas hasta aquel momento estables ${ }^{8}$.

Como última inflexión, Svampa describe una nueva etapa a partir de los sucesos ocurridos durante los primeros años del siglo XXI, en donde, luego de una visible desnaturalización de la relación entre globalización y neoliberalismo, se inicia un ciclo anti-neoliberal. Este ciclo tiene como consecuencia una rehabilitación del uso del concepto de "movimiento social". Al respecto, Svampa explica que la rehabilitación es necesaria en la medida en que se necesita un análisis en otros niveles de la acción que no se contemplan en el estudio de las protestas sociales, tales como las dimensiones cultural e ideológica, relacionados con los procesos de construcción de identidades colectivas.

En estas transiciones, en principio, las nociones de "movimiento social" y de "protesta social" parecen no contraponerse necesariamente. En este caso, no se trata de nociones completamente antagónicas -en el sentido de que intenten dar cuenta del mismo fenómeno desde puntos de vista enfrentados-, sino paralelas. Cada una se usaría para aproximarse a diferentes momentos y aspectos de la acción colectiva. En ese sentido, para Schuster, la noción de "protesta social" se presenta dentro del problema genérico de la acción colectiva, es decir, en la dilucidación de los elementos que permitirían la transición de una acción individual a una acción colectiva; mientras que la noción de "movimientos sociales" se interesa en la cuestión de la constitu- ción de la identidad dentro de los movimientos. En todo caso, a pesar de la diferenciación, en el contexto particular de Argentina, en cuanto el estudio de la acción colectiva, tras los varios cambios sociales y políticos, se ha privilegiado una u otra unidad de análisis ${ }^{9}$.

El problema que señala Alberto Melucci, al hablar de las teorías de los años setenta sobre la acción colectiva, parece repetirse en Argentina: "Los dos puntos de vista no son irreconciliables. Cada uno de ellos es legítimo en sus límites, pero ambos, desgraciadamente, con frecuencia y tal vez implícitamente, se toman como una explicación global” (1999, p. 37).

Ante el escenario anterior, Svampa reivindica el actual punto de vista centrado en los "movimientos sociales", a la vez que abandona cualquier pretensión de un antagonismo con el punto de vista centrado en la "protesta social". Inclusive, aboga por tender ciertos puentes entre los dos; aunque no olvida mostrar que la noción de "movimientos sociales" sí tiene elementos que le permiten dar cuenta de situaciones que, desde la perspectiva de Schuster, son característicos del momento actual y que solo se podrían explicar desde la noción de "protesta social".

Por su parte, en un primer momento, Schuster describe los problemas que conllevaría la caracterización de la movilización social en términos de "movimientos sociales" y señala el problema que representa el ampliar el uso de la noción a cualquier forma de acción colectiva. En ese entonces abogaba por un modelo único y omnicomprensivo que contaba con un consenso "bastante" extenso y en el que confluian diversos estudios en un gran marco común: la agenda clásica (Schuster y Pereyra, 2001, p. 47). En un escrito posterior, Schuster parece proponer, nuevamente, la necesidad de una aproximación entre los dos enfoques, ya que la acción colectiva no podría explicarse solo desde la identidad -emparentada con la noción

8 En el contexto norteamericano, los orígenes de la agenda clásica para el estudio de los movimientos sociales se pueden rastrear hasta los años sesenta con las investigaciones que buscaban llenar los vacíos del enfoque funcionalista en sus estudios sobre el comportamiento colectivo (Delgado, 2007, pp. 53-54). Asimismo, dada la proliferación de movimientos sociales y las revoluciones, su estudio, "[...] tanto en Europa como en Estados Unidos [...] se ha convertido en una industria en expansión en el ámbito de las ciencias sociales" (McAdam, McCarthy y Zald, 1999, p. 22). De aquellas investigaciones surgieron tres paradigmas: la movilización de recursos, el proceso político y los procesos enmarcadores. Finalmente, tras las críticas mutuas, las tres perspectivas convergieron hacia mediados de la década de los noventa y, sumadas a desarrollos más contemporáneos, conformaron el ahora paradigma dominante en este campo.

$9 \quad$ En este escrito se habla de una diferenciación en el contexto argentino; no obstante, se debe anotar que, mientras Svampa ubica la diferencia en un nivel latinoamericano, Schuster, en un primer momento, habla de Argentina y, en un segundo momento, se refiere a una diferenciación en los estudios sobre la acción colectiva sin llegar a ubicar la diferencia territorialmente. 
de "movimientos sociales"-, que no da cuenta de los episodios de acción colectiva fragmentarios, y tampoco únicamente desde la racionalidad emparentada con la noción de "protesta social"ya que no se puede llegar a la conclusión de que las acciones colectivas solamente provienen de una asociación esporádica producto de un cálculo como resultado de la racionalidad estratégica. En todo caso, en aquel momento, llega a una conclusión más matizada, sin comprometerse explícitamente con algún marco teórico (Schuster, 2005, p. 46).

Ciertamente, el debate entre las dos posiciones presenta de primera mano la experiencia latinoamericana enfrentada a una "orfandad teórica" (Gohn, 1997, p. 218, citado en Svampa, 2009, p. 3). A partir de las referencias europeas y norteamericanas, se intenta explicar lo que ocurre en cada país de la región, pero estos marcos son constantemente puestos a prueba por los hechos; lo que redunda en el cambio de un modelo explicativo a otro. Schuster y Svampa nos muestran cómo, a finales de los años setenta, se da una crisis del concepto de clase como explicación de la acción colectiva; sin embargo, Svampa señala que, a pesar del uso de conceptos relacionados con la "clase" o el "movimiento obrero", en Argentina -y, en una extensión del análisis que hace la propia autora, también en América Latinano se podía hablar propiamente a partir de ellos, sino que lo que se designaba bajo esas nociones correspondía, más bien, a las "clases populares" -indígenas, campesinos, trabajadores informales y clase obrera urbana- (2009, p. 2). En un paso posterior, se describe cómo el uso de la categoría de "nuevos movimientos sociales" resultaba algo engañoso, ya que varios de los movimientos analizados poco tenían de nuevo. Para finalizar, la crisis de los movimientos sociales en los años noventa llevó a que las miradas se hicieran desde el marco de referencia norteamericano.

Con todo, si bien el debate es heredero de las discusiones entre la tradición norteamericana y la tradición europea, parece no ser llevado en sus mismos términos. Su discusión no se trata entonces de averiguar cuál es el paradigma que permite una mejor comprensión de la acción colectiva y los movimientos sociales de cualquier parte del mundo, sino de cuál permite una mejor aproximación al contexto específico de América Latina y, concretamente, a cada una de las realidades nacionales. Así pues, la discusión no se enfoca en la fundamentación de cada punto de vista, pero sí en su pertinencia. Por ejemplo, un autor como James M. Jasper —que, en sus palabras, se considera como parte integral de los "paradigmas creados por los hombres blancos de Europa y Estados Unidos" (2012, p. 38)— presenta la discusión entre las grandes teorías en términos de su fundamentación: ya sea si la imagen y la explicación de la acción humana es completa o no, si es un enfoque macrosocial y microsocial y cuál es el mejor, etc. Mientras tanto, Schuster y Svampa parecen no enfocarse en aquellas distinciones, sino en lo que puede observarse en Argentina y en América Latina, hasta el punto de discutir el papel del intelectual respecto a los movimientos sociales o los procesos emancipatorios que representan -elementos que ni siquiera son nombrados por Jasper-.

Por otra parte, como otra característica de los estudios de la acción colectiva en Argentina y, en opinión de Svampa, en toda América Latina, se tiene que, a pesar de las transiciones conceptuales, la acción colectiva se ha caracterizado como antagónica $\mathrm{y}$, especialmente, como emancipadora. La autora recuerda que en la región ha prevalecido una lectura de los movimientos sociales en un sentido fuerte, en donde estos se convierten en actores que cuestionan la lógica de la dominación y buscan una emancipación. Asimismo, señala que, paralelamente, en los últimos años, los cambios ocurridos han traído consigo un retorno de algunos términos tales como "antimperialismo", "descolonización" o "emancipación”, expulsados del lenguaje político y de las academias en épocas pasadas.

De un modo similar, Schuster señala una relación directa entre la acción colectiva y la ciudadanía: en un mundo donde la última está puesta en cuestión, la primera entabla una disputa por ampliarla o instituirla. Desde su perspectiva, la ciudadanía, entendida como un conjunto de derechos que corresponden a los habitantes de un Estado, se ha visto comprometida por una creciente desigualdad que tiene, como consecuencia directa, la iniquidad en el acceso a los derechos. Ante la situación, la acción colectiva se lleva a cabo en el espacio público para reclamar al Estado garantías básicas, una ampliación de los derechos y la posibilidad de ejercerlos.

\section{Supuestos ontológicos y epistemológicos de la noción de protesta social}

Dentro del debate anterior, la acción colectiva es definida por Schuster como "[...] cualquier acción 
que requiere de la participación cooperativa de al menos dos individuos para su realización" (2005, p. 43). En su definición más general, se necesita que se lleven a cabo acciones individuales paralelas que permitan la realización del objetivo final de la acción colectiva. Concretamente, esta puede reflejarse en el pago de impuestos, en una marcha de aficionados a un equipo de fútbol, en una protesta social, en una revolución, etc.

Los estudios de la acción colectiva pasan, en un primer momento, por la pregunta por cómo es posible la acción colectiva. Para Schuster, en su intento de respuesta al problema, la mayoría de las aproximaciones anteriores a la introducción de la noción de movimiento social tendieron a un determinismo de la acción humana. De forma general, en el determinismo se parte de la suposición de que la acción colectiva es algo dado de por sí; esto es, que los individuos solo son el recipiente de propiedades colectivas y la acción social es un producto necesario de las condiciones estructurales.

Dentro de la crítica al determinismo, un autor como Alberto Melucci (1999, p. 36) identifica que varios enfoques usados anteriormente en el estudio de la acción colectiva se mantuvieron entre dos dualismos: en primer lugar, el presentado en términos de aislamiento/solidaridad y, en segundo lugar, en términos de estructura/motivación. El primer dualismo era representado por las teorías del comportamiento colectivo y de la sociedad de masas, que veían a la acción colectiva como producto de la crisis económica y de la desintegración social, y por las aproximaciones derivadas del marxismo, que identificaban la acción colectiva como una expresión de intereses compartidos dentro de una situación estructural común - por ejemplo, como condición de clase- - En el segundo dualismo, la acción colectiva era un producto de la lógica del sistema - contexto socioeconómico- o el resultado de creencias personales -ideología y valores-. Dentro de su determinismo, estas teorías no pudieron explicar la transición de las condiciones sociales a la acción colectiva y tampoco el conflicto, al que terminaron viendo como una reacción patológica y marginal.

Contrarios al determinismo, varios marcos teóricos reafirman la idea de que la acción humana es un factor constitutivo de la realidad. Específicamente, la acción colectiva y el conflicto que presenta no son, necesariamente, una reacción. En el caso de Melucci, la acción colectiva se constituye en una construcción social que necesita de una inversión organizativa; en otras palabras, los seres humanos definen su acción operando dentro de un campo sistémico de posibilidades y límites. En el caso de un autor como Sidney Tarrow, el problema de la acción colectiva no es tanto como se pasa de una acción individual a una acción colectiva, ya que esta última está siempre presente, sino que es un problema de carácter social: "como coordinar a poblaciones desorganizadas, autónomas y dispersas de cara a una acción común y mantenida” (1997, p. 33).

Por su parte, en sus estudios sobre la protesta social, Schuster también comparte la importancia de la acción humana en la movilización social y le otorga una preponderancia en la medida en que existe una contingencia en toda construcción social que la hace posible.

Para ubicar lo anterior, Schuster parte de la ontología política de Laclau ${ }^{10}$, en donde se reconoce que el concepto de totalidad social ha entrado en crisis. La idea moderna de que cada configuración social es un momento de transición hacia una forma más alta de organización se ha encontrado con una nueva conciencia de los límites. Esta constatación se traduce en la comprobación de una premisa que niega cualquier determinismo: no es posible establecer un principio subyacente, en cuanto esencia inmutable o fundamento, en la base de la vida social y sus variaciones. Para Laclau, todos los intentos de crear una sociedad, en cuanto un orden cerrado de la vida social, se enfrentan a la infinitud de lo social y:

[...] [al] hecho de que todo sistema estructural es limitado, que está siempre rodeado por un 'exceso de sentido' que él es incapaz de dominar y que, en consecuencia, la 'sociedad' como objeto unitario e inteligible que funda sus procesos parciales, es una imposibilidad (Laclau, 1993a, p. 104).

En última instancia, la imposibilidad de un fundamento de lo social se transforma en una "esencia negativa" que coloca en el trasfondo la posibilidad siempre latente de intentos —nunca

10 Schuster también reconoce la influencia de A. Giddens, P. Bourdieu y N. Luhman. A pesar de este reconocimiento, se debe notar la influencia principal de Laclau; más aún, si se tiene en cuenta que Schuster adelantó sus estudios doctorales en Teoría Política y Social en la universidad donde Laclau era profesor de Teoría Política: la Universidad de Essex. Por otra parte, la influencia de la ontología de Laclau también se ubica en los estudios que parten desde la noción de movimientos sociales, como lo señala Svampa (2009, p. 11). 
acabados- de fundamentación y de creación de un nuevo orden que acabe con las diferencias.

En este marco ontológico la noción de contingencia es central. Ante todo, su radicalidad no remite a una indeterminación pura y absoluta o a la imposibilidad de toda coherencia dentro de cualquier objetividad social. Antes bien, la contingencia significa que, ante una objetividad social que pretende ser plena y cerrada, siempre puede existir un elemento de impureza que deforma e impide la constitución plena y que se puede constituir en una amenaza para su existencia. Coexisten entonces dos momentos que se convierten en la condición de posibilidad de cualquier amenaza: la plenitud de una objetividad y la imposibilidad de la misma. Tal coexistencia impide que se pueda hablar de una objetividad pura o de una contingencia total: "Lo que encontramos [...] es siempre una situación limitada y determinada en la que la objetividad se constituye parcialmente y es también parcialmente amenazada; en la que las fronteras entre lo contingente y lo necesario se desplazan constantemente" (Laclau, 1993b, p. 44).

Desde la ontología de Laclau, Schuster concluye que, al existir una falla irremediable de estructuración de lo social, se abren "[...] las dimensiones de contingencia, indeterminación y complejidad en la construcción y funcionamiento de dicho orden" (2005, p. 77). De esta manera, frente a la idea de que la sociedad puede ser una totalidad homogénea, unitaria, coherente y armónica, a partir de la premisa del fundamento "negativo" de lo social, Schuster establece que lo constitutivo de la realidad social es la diversidad, el conflicto y la dislocación.

Dentro de esta realidad, la protesta sería una muestra arquetípica de la ruptura de un orden establecido y de la ausencia de fundamentación de lo social. La protesta social se propone como una novedad, en cuanto que es una ruptura con la serie de interacciones sociales que existían con anterioridad. Schuster califica esta novedad como un salto político en el que se tiende un puente entre unas condiciones sociales específicas y un escenario nuevo, en el que los sujetos se implican de una manera nueva.

Por otra parte, la noción de contingencia también es central al momento de interrogar sobre lo social. La ontología aquí planteada tiene un corolario epistemológico: existe la imposibilidad de hallar un principio de inteligibilidad de la sociedad que se constituya en un objeto reconocible de conocimiento. Así pues, este corolario se opone a una de las formas de acercarse a lo social, en donde se parte de una visión objetivista y positiva que niega la contingencia:

El análisis intentará develar, más allá de la conciencia de los actores sociales y de las formas fenomenales en las que su acción se realiza, una racionalidad que se establece en el nivel de las esencias. [...] Entender la historia consiste, pues, en una operación de reconocimiento: de lo que se trata es de identificar, en los actores empíricos, los actores esenciales que en ellos se encarnan y cuya identidad fundamental se conoce de antemano (Laclau, 1993b, p. 38).

Antes bien, una contingencia radical significa que, si lo social no responde a un orden predeterminado, las formas que pueda tomar nunca están determinadas de antemano.

Con todo, esta indeterminación no significa una relación externa y aleatoria entre lo social y sus condiciones; por el contrario, existen en un todo inseparable. De esta forma, para Laclau, lo social, en su indeterminación, depende enteramente de unas condiciones de existencia, por lo que la relación entre lo social y sus condiciones es absolutamente necesaria. En otras palabras, toda objetividad de lo social es concreta y depende de unas condiciones factuales de existencia, no de una especie de esencia.

Cuando se tiene en cuenta esta contingencia radical, la interrogación sobre lo social se modifica:

Hay así una historización de las categorías del análisis social que, al ligar la unidad entre los componentes de un objeto a condiciones de existencia contingentes y específicas, introduce una inestabilidad esencial en las relaciones entre dichos componentes. Mientras que el primer tipo - objetivista- de interrogación de lo social trata de buscar personajes esenciales por detrás de la especificidad histórica, el segundo tipo de interrogación se mueve en la dirección opuesta: debilitando el límite de esencia a través de la contextualización radical de todo objeto (Laclau, 1993b, p. 39).

A partir de este marco ontológico, Schuster parte de la suposición de que, dentro de los estudios de la acción colectiva, la protesta social, como objeto de investigación, posee una contingencia radical. Desde el terreno de las prácticas sociales 
sedimentadas, puede surgir, de manera contingente, una protesta, a partir de unas condiciones que nunca antes habian generado una. Se puede dar también lo contrario: de condiciones que habían generado una protesta, no darse nada en una situación similar. Igualmente, no todas las protestas se transforman en un movimiento, bien pueden manifestarse como un hecho aislado sin futuro.

Ciertamente, las protestas sociales se pueden investigary explicar desde diversas variables, de tal forma que se establezcan las condiciones que hicieron posible la protesta y las razones por las que se llevó a cabo; no obstante, no se trata de variables necesarias, sino que se encuadran en la contingencia radical de toda formación social. En otras palabras, la presencia de ciertas variables específicas no asegura de antemano que se presente una acción de protesta. En últimas, se sabe que ciertas variables posiblemente causaron una protesta solo porque esta se llevó a cabo.

Aquí, Schuster retoma la diferencia entre fundamentación y explicación. Mientras que en la primera se ubicarían ciertas variables como necesarias - el reconocimiento de los actores esenciales- para llegar al corolario de que en la presencia de tales variables siempre habrá una protesta; en la segunda, de lo que se trata, es de contextualizar cada protesta, para atribuirle cierto sentido dentro de un marco conceptual: "La función de la explicación en la reconstrucción de una protesta es la de restaurar la trama de sentido entre la protesta misma y el resto del acontecer social” (2005, p. 55).

En todo caso, Schuster no lleva al extremo de una relativización radical a toda explicación. El margen de interpretación estará restringido por la capacidad explicativa que tenga cada marco conceptual, en cuanto qué tan coherente, relevante y significativo es su relato. Aunque, en este punto, el autor no establece específicamente quién o cómo se establece lo coherente, relevante o significativo que pueda llegar a ser una interpretación. La exposición se limita a caracterizar esta explicación como "científica" - en contraposición a la fundamentación filosófica-, que significa construir un relato en un todo sistémico - a la manera de una imagen en perspectiva de un momento- colocando en ciertas posiciones los datos - en un primer o segundo plano- de acuerdo con la matriz de interpretación que establezca una teoría.

Llegado a este punto, aparte de la pregunta por cómo es posible la acción, los estudios de la acción colectiva pasan, en un segundo momento, por una diferenciación de los tipos de acciones. En un primer caso, dentro de los marcos que partían de cierto determinismo, no se podía establecer una diferencia clara entre las orientaciones que puede tener la acción colectiva, ya que todas ellas obedecían únicamente a factores estructurales, ya sea como expresión de estos o como reacción a sus fallas:

En esta lógica no hay espacio para una capacidad conflictual que surja del interior del sistema, para una acción social que no sea una simple respuesta respectiva a las disfunciones del sistema. El conflicto se eleva siempre al rango de efecto secundario de los procesos de adaptación. De estos presupuestos teóricos deriva también la imposibilidad de introducir distinciones significativas entre las varias formas de acción colectiva (Melucci, 1999, pp. 32-33).

Melucci sostiene que, si se quiere ir más allá de simplemente señalar un hecho como fenómeno colectivo genérico, se deben introducir ciertas distinciones analíticas dentro del amplio rango de acciones colectivas. En el caso particular del autor, se identifican por lo menos tres: si el fenómeno colectivo implica solidaridad o es una simple agregación de individualidades; si el fenómeno colectivo implica la presencia de un conflicto o emerge de un consenso, y, por último, si el fenómeno colectivo trasgrede o no los límites del sistema social. Desde su experiencia investigativa, el autor sostiene que ese conjunto de distinciones analíticas permite separar las diferentes orientaciones de la acción colectiva, que pueden presentarse o no en diversas combinaciones dentro de un fenómeno colectivo en particular. Verbigracia, la definición de movimiento social presentaría las siguientes distinciones: a) es basado en la solidaridad, b) desarrolla un conflicto y c) rompe los límites de un sistema. A partir de esos tres elementos, es posible separar lo definido como movimiento social de otros fenómenos colectivos tales como la delincuencia, las reivindicaciones organizadas, el comportamiento agregado de masas, etc.

Desde la tradición norteamericana, Sidney Tarrow afirma que la acción colectiva no es una categoría abstracta que se pueda aplicar sin más a todo tipo de fenómeno colectivo (1997, p. 20). La acción colectiva, generalmente, se produce en el marco de las instituciones por parte de grupos constituidos con objetivos considerados normales; 
pero, en ocasiones, se convierte en contenciosa cuando es usada por personas que no pueden acceder a las instituciones, que llevan consigo reivindicaciones novedosas $\mathrm{o}$ no aceptadas $\mathrm{y}$ pueden constituir una amenaza fundamental para otros. De esta forma, puede que el problema de la participación colectiva en una acción sea generalizado, pero para explicar los diferentes tipos de fenómenos colectivos se debe tener en cuenta que los recursos y problemas de coordinación no son iguales en todos los ámbitos. En su particularidad, los movimientos sociales llevan a cabo una acción colectiva contenciosa histórica y sociológicamente distintiva, que no se puede comparar con una acción colectiva no contenciosa, así se tenga como objetivo el conseguir una mayor capacidad explicativa.

Por su parte, Schuster no define a profundidad lo que puede ser un "movimiento social", pero sí establece una diferenciación de este con la "protesta social" en términos de la continuidad o no de las identidades y a su extensión espacio-temporal. Específicamente, el sostener una noción como la de "movimiento social" para explicar todas las acciones colectivas puede llevar a dejar de lado otros elementos que no encajan dentro de la continuidad y la extensión espacio-temporal que caracteriza este tipo de acción. Este uso implica una rigidez que no permite describir la variedad de acciones colectivas que tenemos en el presente. Así pues, frente a la rigidez de la noción de "movimientos sociales", se presenta la alternativa de la noción de "protesta social", en donde los protagonistas se asocian y se separan en tiempos relativamente breves, en espacios fuertemente localizados y sin constituir necesariamente identidades que tengan una continuidad más allá del momento de la acción de protesta.

Para ilustrar lo anterior, Schuster parte de la situación argentina tras la caída de la dictadura en 1983, en donde los sindicatos eran el eje de la movilización social, junto con los consolidados movimientos por los derechos humanos. En aquel entonces, para entender la nueva situación, se llevaron a cabo investigaciones inspiradas en los trabajos de Touraine y de Castells, de ahí que se buscara mostrar exclusivamente el surgimiento o la consolidación de "movimientos sociales" (Schuster y Pereyra, 2001, p. 42). En este contexto, el uso de la noción de "movimientos sociales" permitió recuperar aquellas luchas que no se derivaban necesariamente de un conflicto de clases y que no buscaban una transformación de la sociedad.
Ahora bien, en la década de 1990, frente a las reformas neoliberales, los sindicatos, eje central de la movilización en Argentina desde principios del siglo XX, fueron los principales afectados, ya que su oposición a las reformas fue contrarrestada por una aparente bonanza económica (Schuster y Scribano, 2001, p. 18). Asimismo, la eficacia de los medios de reclamo tradicionales, tales como marchas, movilizaciones o huelgas, se vio cuestionada.

A pesar de la inicial recuperación económica y el crecimiento, el modelo socio-económico implementado, poco a poco, dejó ver a los afectados, lo que se tradujo en: aumento en el número y la diversificación de las protestas; multiplicación de las organizaciones de protesta; modificación de los temas y las demandas de las organizaciones y de las acciones de protesta, $\mathrm{y}$, por último, en una aparición de nuevas formas de protestar.

Al final, en vista de estos cambios, Schuster considera que la búsqueda de identidades a partir de la noción de "movimientos sociales" no permite dar cuenta de la diversidad y la fragmentación. Con este enfoque se privilegió la búsqueda de continuidades e identidades que, varias veces, según la evaluación del autor, “[...] existen más en la lente del observador que en la percepción de los propios sujetos implicados en la movilización" (2001, p. 43). La identidad clásica alrededor de los sindicatos o las identidades de los "nuevos movimientos sociales" resultan insuficientes frente a la diversificación creciente.

En la opinión de Schuster, la noción de "protesta social” permitiría un análisis más amplio y genético de la acción colectiva, ya que incluye un número mayor de acciones y eventos que no serían estudiados si se parte de una noción como la de "movimiento social". Asimismo, concentrarse únicamente en los movimientos sociales también impediría recuperar la dimensión política de la acción colectiva y sus múltiples elementos. Tal y como los caracteriza Schuster, los "movimientos sociales" y sus acciones visibilizan un conflicto latente o de larga data, por lo que privilegiar exclusivamente esa noción conlleva una exclusión investigativa de los nuevos antagonismos e identidades que pueden aparecer tras una acción de protesta y sus mecanismos, que únicamente serían estudiados si el observador considera que se han constituido en un "movimiento social". De igual manera, comprender los episodios de protesta en clave de "movimientos sociales" hace que la centralidad de la identidad 
en el tiempo opaque otros elementos que se hacen presentes en la emergencia de una protesta ${ }^{11}$.

En su relación, la noción de "movimiento social" tuvo una preponderancia sobre la noción de "protesta social" debido a la necesidad de plantear un sujeto necesario de la protesta en un intento de fundamentarla desde un punto de vista tanto epistemológico como ontológico. En ese sentido, la protesta se explicaba a partir de un movimiento y, a su vez, este último se explicaba a partir del sistema social. Pero, en la realidad, todo "movimiento social" puede llevar a cabo protestas, pero no toda protesta es llevada a cabo por o deriva en un "movimiento social".

La afirmación anterior no significa que cada protesta sea necesariamente un hecho aislado que no pueda ser analizado en conjunto con otras protestas. De hecho, para Schuster, una protesta adquiere sentido porque se encuentra emparentada con otras acciones de protesta, en cuanto que pueden mostrar rasgos comunes o, por el contrario, romper con ellos y establecer una emergencia. Así pues, si se abandona la idea de que la noción de "movimiento social" es la única clave explicativa de las protestas sociales, el análisis se abre a otras dimensiones, a partir de las cuales se pueden establecer diversas continuidades y rupturas; para llegar, en última instancia, a recortes significativos de la realidad que se constituyen en ejercicios interpretativos.

Al mismo tiempo, los rasgos comunes y las particularidades de las "protestas sociales" dan pie, en los ejercicios interpretativos, a la constitución de lo que Schuster denomina como redes de protesta: "[...] una red es una constatación empírica de la existencia de un tejido invisible pero real entre acciones de protesta en principio diversas" (2005, p. 58). El autor coloca entre las protestas y los "movimientos sociales" este concepto como una especie de eslabón perdido, en cuanto la conformación potencial de un movimiento. En cualquier caso, la concesión no se encuentra exenta de ciertas restricciones. Schuster aclara que, desde un punto de vista epistemológico, las dos nociones corresponden a diferentes instancias de análisis. Por un lado, la noción de redes de protesta se encontraría en una instancia empírico-descriptiva. Por otro lado, la noción de movimiento social sería una instancia teórica-explicativa y, como tal, su aplicación o no, depende de una decisión teórica justificada.
En resumen, Schuster coincide con otros autores en que la acción colectiva no es un mero reflejo de las condiciones estructurales, sino que se trata de un producto de la acción humana en la que confluyen tanto una dimensión intencional como una no intencional, aunque constreñida al sistema de condiciones en las que se lleva a cabo. Desde un punto de vista ontológico, la acción es posible en la medida en que el fundamento de la sociedad es una contingencia que abre la posibilidad a los cambios. Asimismo, como corolario epistemológico, se tiene que toda explicación de una acción colectiva debe ser situada y no derivada de una posible racionalidad que la condicione de antemano.

Dentro del marco anterior, la protesta social se constituiría en una muestra de la contingencia y de la posibilidad de una acción sin fundamento. Sin embargo, esta noción se encuentra con un primer inconveniente al ser establecida como la unidad básica, ya que implica definir toda acción colectiva contenciosa a partir de esta noción.

Para ejemplificar tal movimiento, se puede observar la caracterización de un movimiento social que hace Schuster: "En conjunto, se pide continuidad y extensión espacio-temporal a un sistema o conjunto de acciones para caracterizarlo como movimiento social”. Posteriormente, afirma que aquella caracterización "[...] no parece darse en una cantidad de formas de acción colectiva contemporánea" (2005, p. 46); para terminar estableciendo como alternativa la noción de "protesta social". Así pues, la "rigidez" de la noción de movimientos sociales se contrarresta con la afirmación de que "no solo los movimientos sociales protestan”. Sin embargo, a esta última se le puede contraponer la afirmación de que "no toda acción colectiva contenciosa se lleva a cabo a través de una protesta”. En ese sentido, se puede observar que se repite el problema que Melucci detecta en los enfoques provenientes de la agenda clásica; el cual denomina como una sobrecarga política:

Los conflictos sociales se reducen a la protesta política y a ser vistos como parte de un sistema político. La confrontación con el sistema político y con el Estado es apenas un factor más o menos importante en la acción colectiva. El conflicto frecuentemente puede afectar el mismo modo de producción o la vida cotidiana de las personas (1999, p. 39).

11 Respecto a esta crítica, Svampa responde que, en los procesos de "[...] análisis de la (re)construcción de identidades colectivas, confluyen perspectivas que hacen hincapié en la — no tan novedosa- heterogeneidad y complejidad de las luchas sociales, así como en el carácter contingente y precario de las identidades" (2009, pp. 6-7). 
Esta sobrecarga política hace que autoras como Svampa intenten recuperar los análisis en otros niveles de la acción - cultural, ideológico o subjetivo- que no refieren al sistema político (2009, p. 6). Igualmente, sin ir más lejos, como ejemplo de otro tipo de acción colectiva contenciosa encontramos la noción de creación de un "mundo otro” de Raúl Zibechi (2017).

\section{El marco de análisis de la protesta social}

Dada la indeterminación originaria de la "protesta social", para Schuster no es posible establecer un marco de análisis que pretenda establecer unas leyes en las que antemano se especifique cómo funciona una protesta. Las diferentes propuestas existentes tienden a favorecer algún aspecto en particular - los intereses y la racionalidad, los procesos de constitución de identidad, las condiciones sistémicas, las relaciones de poder, recursos disponibles para la acción, etc.- para aproximarse a cualquier "protesta social”, olvidando su carácter contingente.

En todo caso, como se dijo anteriormente, la contingencia no significa ininteligibilidad. Schuster apunta a solventar dos exigencias: en primer lugar, cualquier marco de análisis, que pretenda ser efectivo, debe contemplar alguna forma casuística de combinación compleja de los diversos factores que se encuentran en los episodios de protesta; en segundo lugar, cualquier marco de análisis debe ser aplicable de modo general y facilitar la comunicación entre investigadores. Concretamente, la propuesta de Schuster plantea un marco que se considera como "[...] un intento de avance complementario y en la misma orientación de las líneas más actuales en estudios de acción colectiva y movimientos sociales" (Schuster, 2005, p. 59).

Schuster propone elaborar tipologías de protesta a partir de cinco variables o unidades de análisis que intentan dar cuenta de los aspectos más relevantes que se pueden encontrar en una protesta social. En síntesis, a través de las variables, pretenden saber, para cada protesta en particular: ¿quién protesta?, ¿cuándo/dónde se protesta?, ¿por qué se protesta?, ¿cómo se protesta? y ¿para qué se protesta? Con las unidades de análisis se busca llegar, en última instancia, a conformar modelos ideales-típicos de la protesta.

Como primera unidad de análisis se encuentra la matriz identitaria —¿quién protesta?
- En ella se busca establecer la historia de los protagonistas de la protesta o de la protesta misma. Aquí, se identifica una conceptualización laclauniana de la identidad en donde existe una doble dimensión: una que se denomina identidad sedimentada, producto de las interacciones rutinizadas; otra que se denomina identidad política, que surge del conflicto, "[...] cuando hay un quiebre entre lo que creo que soy, tengo, merezco, etcétera, y lo que veo" (2005, p. 60). Para cada caso, se debe analizar cuál es que la que se presenta en la protesta.

La segunda dimensión de análisis da cuenta de las condiciones estructurales —¿cuándo/ dónde se protesta? - Dentro de ella se tienen en cuenta las condiciones sociales, económicas, políticas o culturales de los actores y puede incluir también el modo subjetivo en que los actores interpretan tales condiciones. Específicamente, se incluyen también conceptos como la consideración del marco de oportunidades o amenazas; la fortaleza o debilidad del antagonista y de la organización de protesta, o las condiciones de organización que posibilitan la acción.

En cuanto la tercera dimensión de análisis se tiene a la demanda de la acción de protesta¿por qué se protesta? - , que remite a las preguntas de qué pide el sujeto de acción colectiva y cómo lo pide. Dentro de estos elementos se expresan de modo concreto las cuestiones de la identidad y su relación con las condiciones estructurales.

La cuarta dimensión se denomina formato — ¿cómo se protesta? - y parte de lo que Charles Tilly denominó como repertorios de la acción colectiva. $\mathrm{El}$ formato remite a la forma en la que se presenta la protesta - una marcha, una huelga, un plantón, etc. - y se relaciona de manera directa con factores identitarios y racionales. Por una parte, en cuanto la identidad: "[...] la gente no puede emplear rutinas de acción colectiva que desconoce; cada sociedad tiene una reserva de formas familiares de acción [...] que se convierten en aspectos habituales de su interacción" (Tarrow, 1997, p. 51). Asimismo, Schuster relaciona la cuestión identitaria del formato con el concepto, usado en la sociología política norteamericana, de enmarcamiento cultural: como problema social, en la acción colectiva, se llevan a cabo procesos colectivos de interpretación, atribución y construcción social, que llevan de las oportunidades a la acción (Jasper, 2012, p. 28; McAdam, Tarrow y Tilly, 2004, p. 41; Tarrow, 1997, pp. 57-58). En cuanto la racionalidad, se refiere a la posibilidad de tomar 
una decisión estratégica que busque el modo más eficaz de actuar; aunque, dada la indeterminación constitutiva de la protesta, este proceso de decisión no se debe establecer necesariamente como una acción previa, ya que se puede dar durante la misma protesta o estar implícito y ser encontrado en las reconstrucciones de lo sucedido.

La última dimensión, relacionada con el impacto político de las protestas, se denomina performatividad - ¿para qué se protesta? - A partir de algunas herramientas provenientes de la metodología del análisis del discurso, Schuster propone analizar algunos aspectos de la producción discursiva de los actores sociales que participan en la protesta. Igualmente, se busca establecer los resultados de la acción en cuanto: su efecto estratégico, es decir, la relación entre la demanda y su posible satisfacción al final de la protesta; su efecto institucional — crisis política, caída de un gobierno o conformación de un nuevo espacio político-, y su efecto performativo, esto es, la capacidad para instalar en el debate político un tema que antes no era cuestionado.

Finalmente, a partir de estas cinco dimensiones, Schuster propone ir desde las protestas sociales, pasando por las redes de protesta, hasta la posibilidad de establecer — desde un punto de vista analítico- movimientos sociales.

Como unidad de análisis básica se tiene:

[...] la noción de protesta social se refiere a los acontecimientos visibles de acción pública contenciosa de un colectivo, orientados al sostenimiento de una demanda - en general con referencia directa o indirecta al Estado-. En este sentido, cabe remarcar que el concepto se limita a partir de su carácter contencioso e intencional, por un lado, y de su visibilidad pública por el otro. Las manifestaciones, las concentraciones o movilizaciones públicas son ejemplos de tipos de protesta aprehendidos desde este punto de vista (Schuster, 2005, p. 56; Schuster et al., 2006, pp. 13-14; Schuster y Pereyra, 2001, pp. 47-48). ${ }^{12}$
Desde la definición anterior, Schuster plantea un problema respecto a qué se debe tomar como una protesta - la unidad de la protesta-, ya que, como acontecimiento, una protesta puede ser considerada como cada acción directa que se presente -dentro de un paro, por ejemplo, cada actividad podría ser considerada como una protesta-. Sin embargo, se señala una apuesta teórica que coloca, en el lugar principal, a la acción humana o el carácter subjetivo. En ese sentido, se privilegia el carácter activo de un sujeto que puede definir cursos de acción, por lo que se considera que la unidad del sujeto es lo que le da la unidad a la acción de protesta - dentro de un paro, cada actividad es llevada por un mismo sujeto, por lo que se puede considerar el paro como un solo acontecimiento de protesta-.

A partir de las cinco dimensiones de análisis se pretende encontrar continuidades y rupturas entre los diversos acontecimientos para llegar a plantear redes de protesta $\mathrm{y}$, en última instancia, a la identificación de un movimiento social. Para ello, Schuster se apoya en la identidad que se puede observar en las acciones, las organizaciones y las demandas. Tal identidad es diferente a lo que él denomina como matriz identitaria y, más bien, parte desde un concepto filosófico como el de mismidad ${ }^{13}$. Se infiere que, a partir de las definiciones empíricas, se puede llegar a establecer la existencia de un movimiento; en otras palabras, a partir de los acontecimientos concretos de protesta y de las dimensiones de análisis se plantea la posibilidad de hallar una identidad — mismidadde las acciones, las demandas y las organizaciones.

Ahora bien, a pesar de la posibilidad teórica de encontrar un movimiento social, en definitiva, la noción de protesta social tiende a tener como resultado análisis empíricos-descriptivos y a dejar de lado los análisis teóricos-explicativos. El marco de análisis propuesto plantea cinco unidades de análisis que intentan integrar conceptos objetivos y subjetivos y estructurales y agenciales que tienen como fin el comprender y explicar

12 En la aplicación práctica, en el trabajo del GEPSAC también se manejó otra definición de una acción de protesta, a partir de la agenda clásica: "Se definió una acción de protesta como un evento de carácter contencioso producido por un actor social colectivo que implica movilización de recursos. Considerábamos, entonces, como acción unitaria aquella que se deriva de un esfuerzo único de organización y movilización de recursos, independientemente de la continuidad de la acción" (Schuster et al., 2006, p. 7). En la última presentación de los resultados de la investigación, se partió de esta última definición (Pereyra et al., 2017, p. 578).

13 En un sentido ontológico, la identidad así reconocida se refiere a la dimensión del ser que perdura a través del tiempo, entendido como un núcleo de permanencia que garantiza la continuidad ininterrumpida entre el primer y último estadio de lo que se llama la "misma" cosa (Blanco Ilari, 2006)凶. 
la forma privilegiada de acción colectiva. En su indeterminación y contingencia, los episodios de protesta social analizados se toman como datos y, por lo tanto, como unidades empíricas que pueden ser desagregadas en unidades más pequeñas - a partir de las cinco unidades de análisis- con el objetivo de agregarlas o desagregarlas para llegar, en última instancia, a ciertas conclusiones descriptivas ${ }^{14}$. De esta manera, se llega a responder a la pregunta ¿qué pasó?, sin llegar a abordar la pregunta ¿por qué pasó? Concretamente, como se verá más adelante, se llega a diversas descripciones y taxonomías acerca de la realidad de la protesta social en Argentina sin explicar por qué se dio aquella realidad y no otra.

\section{El paso de la teoria a la práctica}

En la aplicación concreta de la noción de protesta social, elGEPSACbuscaindagarlastransformaciones de la protesta social en Argentina. Para ello, parten de una mirada de largo plazo que busca trascender el estudio de algunas organizaciones o situaciones conflictivas particulares y llegar a un entendimiento sobre la presencia y el impacto de la protesta en la política argentina. Para tal fin, el grupo plantea una investigación empírica en la que se confeccionaría un mapa de protestas exhaustivo en donde se analizarían todas las acciones de protestas, producidas en la Argentina desde 1989 ${ }^{15}$, a partir de las cinco dimensiones descritas en el acápite anterior. El trabajo comenzaría en 1998 y contaría, como fuente de información básica, con las noticias sobre protestas sociales consignadas en los periódicos nacionales en el periodo 19891998. En estas fuentes se esperaba identificar una serie de variables que combinaban datos sobre las acciones de protesta y sobre el medio periodístico (Schuster et al., 2006, p. 12).

En esta primera etapa de la investigación, el grupo partía del hecho de que continuamente se presentaban reclamos al Estado, por un lado, por garantías de libertad, seguridad ciudadana, igualdad ante la ley y no discriminación y, por el otro, por derechos elementales de subsistencia, salud, educación y ambientales. En consecuencia, a partir de la diferencia en los reclamos, se plantearon tres supuestos de investigación: la primera clase de reclamos se estructuraban alrededor de la noción de regeneración del espacio público, la segunda clase se explicaba a partir del concepto de interés $\mathrm{y}$, por último, entre las dos clases de protesta, la cívica y la corporativa, el GEPSAC identificaba que la primera era la que tendía a primar en el periodo estudiado. Finalmente, dada la primacía de la protesta cívica, se plantearon una serie de hipótesis que entrecruzaban los estudios de la acción colectiva con las discusiones alrededor del concepto de ciudadanía y que pretendían establecer el modo en que la noción de ciudadanía se encontraba en las acciones de protesta argentinas.

Entre 1999 y 2001 se realizó la primera recolección de la información para el periodo ya extendido hasta el año de 1999. Con todo, mientras se desarrollaba el trabajo de esta primera etapa, el grupo decidió explorar otra serie de hipótesis que, desde su perspectiva, maximizaban las potencialidades de su trabajo descriptivo: la primera se refiere a la relación entre los cambios sociopolíticos y las transformaciones en los sujetos de las protestas y el impacto político de estas últimas, la segunda afirma el carácter ahora fragmentario de las protestas y, la última, da cuenta del impacto político de las protestas, las cuales, aunque no buscan modificar el sistema social establecido, sí tienen un gran impacto en relación con la emergencia de nuevos actores, problemas y estrategias. Llegados a este punto, en un segundo momento del proyecto ${ }^{16}$, la cuestión de la ciudadanía fue relegada para concentrarse en el vínculo existente entre las protestas sociales y las transformaciones sociopolíticas que se presentaron, entre 1989 y 2003, en Argentina. Para ese momento, la ampliación se consideró importante debido a los acontecimientos que siguieron a la crisis de 2001 en Argentina.

Aparte del cambio en las hipótesis de trabajo, el GEPSAC enfrentó una serie de problemas metodológicos. Las fuentes de información pronto

14 Concretamente, en la aplicación del marco teórico y la metodología, la investigación de la GEPSAC, por ejemplo, a partir de la dimensión de matriz identitaria encontró que, entre 1989 y 2003, los sindicatos fueron los que llevaron a cabo el mayor número de protestas; a partir de la dimensión de la demanda, encontraron que el 37\% de las demandas fueron laborales; en la dimensión del formato encontraron que el más utilizado fue la movilización y que las típicas formas de protesta sindicales — paros y huelgas_ cedieron su importancia a los cortes de ruta; entre otros hallazgos (Pereyra et al., 2017; Schuster et al., 2006).

15 Acerca de las razones para escoger este año como el inicial, ver la nota a pie de página 6.

16 Esta segunda etapa de investigación se llevó a cabo entre 2001 y 2003. 
se mostraron cortas para las necesidades del proyecto y se tomó la decisión de concentrar el trabajo en aquellas dimensiones cuya información se encontraba más a la mano; específicamente, las dimensiones de identidad - acotada como organización-, formato y demanda, para dejar de lado la dimensión de performatividad.

En su informe final (Schuster et al., 2006), el GEPSAC describe cómo la aplicación concreta del método de análisis de las protestas sociales se encontró con ciertos obstáculos prácticos, lo que conllevó a cierta acotación de sus pretensiones. Sin embargo, el problema no se encuentra tanto en el trabajo informativo que llevan a cabo los diarios, sino en la misma unidad de análisis: el acontecimiento de protesta. La operativización de la noción de protesta social adolece de un segundo problema ya planteado por Melucci: la unidad de análisis escogida tiende a favorecer la escena pública y excluye la red de relaciones que se encuentra antes, durante y después de los sucesos (1999, p. 61). En este caso, la fuente desde donde se recoge la información del acontecimiento - en este caso los informes de prensa - se constituye, más bien, en una ayuda para llevar a cabo la exclusión.

El GEPSAC plantea que los medios de comunicación se encuentran sometidos a ciertas reglas de juego que son consecuencia de ciertas expectativas sociales acerca de su función como vehículos y agentes en la circulación de la información. En ese sentido, tales reglas de juego reducirían las posibilidades de manipular la información, por lo que, en última instancia, “[...] los medios más que actores con intereses políticos definidos se constituyen en escenarios donde los conflictos sociales se expresan buscando legitimarse frente a la opinión pública nacional" (Schuster et al., 2006, p. 20). Sin embargo, precisamente, en cuanto escenario, los sucesos presentados son un resultado objetivizado que da cuenta de ciertos elementos y no otros; no tanto como resultado de una manipulación de la información, sino como producto de las expectativas de los sujetos de la protesta que, en su afán de legitimidad, consciente o inconscientemente, se presentan de cierta forma resaltando u ocultando diversos elementos.

Como consecuencia del problema anterior, se simplificó la investigación y el proyecto tomó una forma netamente descriptiva y cuantitativa.
En esta última interpretación del proyecto, una de las metas era "[...] recuperar la importancia de la dimensión de la acción de los movimientos y emplear un tipo de análisis en el que la acción se vuelva independiente, en cierta medida, de los procesos sociales que en última instancia la hacen posible" (Pereyra et al., 2017, p. 578). Aquí, la recuperación de la dimensión de la acción no se traduce en un análisis de las organizaciones argentinas sino en un análisis de lo que denominaron como el registro público de la actividad de las organizaciones. Dicho registro, en cuanto historia de las protestas, se presenta como relativamente independiente de la historia de las organizaciones. Para el GEPSAC, en última instancia, la visibilidad y el impacto político de la movilización dependen más de las acciones de protesta que de las trayectorias organizacionales y su peso relativo en el sistema político.

En términos generales, a partir de los datos, el GEPSAC concluye que la fragmentación de las protestas, en términos de la singularización de demandas y la multiplicación de actores, parece no ser una constante y una tendencia que se mantenga en el tiempo. En cuanto la singularización de las demandas, se presentó un pasaje desde reivindicaciones particularistas a reivindicaciones de carácter más general. En ese sentido, la hipótesis de que los actores no buscaban una transformación del sistema social no parece ser cierta.

Respecto a la multiplicación de los actores, la conclusión se torna algo confusa:

Como contracara de la retirada de algunos sectores sindicales y políticos del escenario de la protesta social, nuevos sujetos han adquirido una relevancia fundamental. Algunos de ellos tienen una larga historia vinculada a la movilización social, como es el caso de los vecinos, las organizaciones de derechos humanos y los estudiantes, pero otras tienen un carácter más novedoso como es el caso de los familiares de víctimas o el de los piqueteros (Schuster et al., 2006, p. 63) ${ }^{17}$.

Por el contrario, dada la larga historia vinculada a la movilización social, no es posible sostener que solamente nuevos sujetos han adquirido una relevancia, excepto por los familiares de víctimas y el importante caso de los piqueteros.

Por último, se pone en duda la afirmación de que los cambios en las sociedades actuales 
producen individuación, riesgo y desmovilización. El alto nivel de movilización social en el periodo estudiado en Argentina, parece demostrar que más bien se presentó "[...] un desacople entre beligerancia social y sistema político; el tránsito de una representación en el poder a una representación y constitución de actores y demandas contra el poder" (Schuster et al., 2006, p. 66).

\section{Conclusiones}

Para dar cuenta de la acción colectiva actual, Schuster y el GEPSAC parten de un rechazo de cualquier determinismo de la acción humana, al plantear como base ontológica la contingencia de lo social. Esta lleva a dos corolarios: en primer lugar, la sociedad no es determinada por un orden preestablecido y, por lo tanto, es susceptible al cambio; y, por otra parte, esta indeterminación conlleva que los fenómenos sociales no puedan ser explicados a partir del establecimiento de una racionalidad que los antecede.

En su planteamiento, Schuster parece privilegiar la noción de protesta social en la medida en que expone, de manera más directa, la contingencia a la que alude. En consecuencia, esta noción se establece como la unidad básica para abordar la acción colectiva contenciosa; sin embargo, tanto a nivel teórico como práctico, pronto se observa que la protesta social se queda corta. La acción colectiva contenciosa no puede ser reducida a la protesta y tampoco se puede comprender solamente a partir de esta.

Contrario a la afirmación, que se hace en la presentación del marco de análisis para las protestas sociales, respecto a que el trabajo debería ser visto como "[...] un intento de avance complementario [...] de las líneas más actuales en estudios de acción colectiva y movimientos sociales" (Schuster, 2005, p. 59), en su aplicación práctica, el GEPSAC explícitamente reconoce que su interés es similar a los análisis de los eventos de protesta provenientes de la sociología política norteamericana (Schuster et al., 2006, p. 17). Asî pues, el trabajo del GEPSAC se enfrenta a una tensión entre su marco de análisis y la aplicación concreta del mismo. El primero se presenta como una forma de dar cuenta de la acción colectiva en la actualidad que permite una inclusión de otros fenómenos sociales diferentes a lo que pueda ser calificado como movimiento social. Mientras que, en lo segundo, se limita al registro público de las actividades de las organizaciones y movimientos.

La tensión quizá se explique a partir de ciertas limitaciones investigativas prácticas limitaciones de información o de tiempo- que se ponen de presente en el informe presentado por el grupo. Sin embargo, su explicación se relaciona más con las limitaciones generales del enfoque que con las limitaciones prácticas que se puedan encontrar en su aplicación. Parafraseando al GEPSAC, el basarse en los análisis de la protesta social de la sociología política norteamericana expone a la noción de protesta social a los dilemas a los que se enfrentan tales enfoques.

Finalmente, ante la "rigidez" de la noción de movimientos sociales, la "flexibilidad" de la noción de protesta social no resulta ser una alternativa viable para la comprensión de la acción colectiva que se presenta actualmente. En definitiva, como se dijo anteriormente, los dos enfoques no se pueden calificar como antagónicos. Los estudios sobre la protesta social no logran ser equivalentes a los estudios de la acción colectiva ni a la noción de movimientos sociales.

Con todo, desde un punto de vista epistemológico, esta conclusión no deja de ser una afirmación descriptiva. Sin embargo, desde un punto de vista político, sí parece llevar a una gran diferenciación. Schuster plantea el problema en términos de las dimensiones del estudio, en donde la neutralidad favorece la explicación, mientras que limita la comprensión, y, a su vez, el compromiso con los actores lleva a lo contrario. Como resultado, se hace un llamado a una vigilancia epistemológica y a una vigilancia ética, con el fin de que los sujetos de la investigación no se conviertan en medios cosificados para el conocimiento $(2005$, p. 71$)$. En la práctica, el acercamiento a la acción colectiva a partir de la noción de protesta social parece favorecer el lado de la explicación. Esta imagen contrasta con la búsqueda del investigador-intelectual comprometido que una autora como Svampa rescata. Dentro de este paradigma, aquella figura es capaz de producir conocimiento que va más allá de los actores y que, al mismo tiempo, los interpela críticamente en su labor de acompañamiento (2009, pp. 12-13).

En Argentina - y quizá en América Latina-, los estudios de la acción colectiva parecen estar fuertemente atravesados por los conflictos sociales de los cuales buscan dar cuenta. Pero tal parece que la escogencia de un enfoque $u$ otro no se constituye 


\section{en una decisión netamente epistemológica, sino que adquiere los tintes de una decisión política.}

\section{Referencias}

Blanco Ilari, J. I. (2006). Promesa e ipseidad: La crítica de Ricoeur al reduccionismo. Revista Latinoamericana de Filosofía, 32(2). Recuperado de http://www.scielo.org.ar/pdf/rlf/v32n2/ v32n2a03.pdf

Blutman, G. (1994). Orden y desorden en la reforma del estado argentino (1989-1992). Ciclos, IV (7), 53-93.

Centro de Estudios Legales y Sociales. (1997). Informe anual sobre la situación de los Derechos Humanos en la Argentina 1996. Buenos Aires: Centro de Estudios Legales y Sociales.

Delgado, J. D. (2007). Perspectivas clásicas y contemporáneas en el estudio de los movimientos sociales: análisis multidimensional del giro hacia la relacionalidad. Revista Colombiana de Sociologia, (28), 47-71.

Gohn, M. da G. (2018). Movimientos sociales en América Latina: marcos teórico-metodológicos. En S. Gómez Obando, C. Moore Torres, y L. Múnera Ruiz (Eds.), Los saberes múltiples y las ciencias sociales y políticas 2 (pp. 65-86). Bogotá: Universidad Nacional de Colombia. Facultad de Derecho, Ciencias Políticas y Sociales. Instituto Unidad de Investigaciones Jurídico Sociales Gerardo Molina (UNIJUS).

Jasper, J. M. (2012). ¿De la estructura a la acción? La teoría de los movimientos sociales después de los grandes paradigmas. Sociológica (México), 27(75), 7-48. Recuperado de http://www.scielo.org. $\mathrm{mx} /$ scielo.php?script=sci arttext\&pi$\underline{\mathrm{d}=\mathrm{S} 0187-01732012000100001 \& \operatorname{lng}=\mathrm{es} \& \mathrm{nr}-}$ $\underline{m}=$ iso\&tlng $=\mathrm{es}$

Laclau, E. (1993a). La imposibilidad de la sociedad. En Nuevas reflexiones sobre la revolución en nuestro tiempo (pp. 103-106). Buenos Aires: Ediciones Nueva Visión.

Laclau, E. (1993b). Nuevas reflexiones sobre la revolución de nuestros tiempos. En Nuevas reflexiones sobre la revolución en nuestro tiempo (pp. 1999). Buenos Aires: Ediciones Nueva Visión.

Leiras, M., Medina, J., y D’Alessandro, M. (2005). La Ciencia Política En Argentina: El camino de la institucionalización dentro y fuera de las Aulas Universitarias. Revista de Ciencia Política, 25(1), 76-91. DOI: https://dx.doi.org/10.4067/ S0718-090X2005000100005

McAdam, D., McCarthy, J. D., y Zald, M. N. (1999). Oportunidades, Estructuras de Movilización y Procesos Enmarcadores: Hacia una Perspectiva Sintética y Comparada de los Movimientos Sociales. Movimientos Sociales: Perspectivas Comparadas. Oportunidades Políticas, Estructuras de Movilización y Marcos Interpretativos Culturales (pp. 21-46). Madrid: Ediciones Istmo.

McAdam, D., Tarrow, S., y Tilly, C. (2004). Dynamics of Contention. Cambridge: Cambridge University Press.

Melucci, A. (1999). Acción colectiva, vida cotidiana y democracia. México: El Colegio de México, Centro de Estudios Sociológicos. Recuperado de: http://www.cervantesvirtual.com/descargaPdf/accion-colectiva-vida-cotidiana-y-democracia-924292/

Pereyra, S., Pérez, G. J., y Schuster, F. L. (2017). Tendencias de la protesta social en Argentina, 19892007. En P. Almeida \& A. Cordero Ulate (Eds.), Movimientos sociales en America Latina: Perspectivas, tendencias y casos (pp. 579-620). Buenos Aires: CLACSO.

Schuster, F. L. (2005). Las protestas sociales y el estudio de la acción colectiva. En F. L. Schuster (Ed.), Tomar la palabra: estudios sobre protesta social y acción colectiva en la Argentina (pp. 4383). Buenos Aires: Prometeo Libros.

Schuster, F. L., y Pereyra, S. (2001). La protesta social en la Argentina democrática: balances y perspectivas de una forma de acción política. En La protesta social en la Argentina: Transformaciones económicas y crisis social en el interior del país (pp. 41-63). Buenos Aires: Alianza Editorial.

Schuster, F. L., Pérez, G. J., Pereyra, S., Armesto, M., Armelino, M., García, A., ... Zipcioglu, P. (2006). Transformaciones de la protesta social en Argentina 1989-2003 (Documento de Trabajo No. 48). Buenos Aires: Instituto de Investigaciones Gino Germani, Facultad de Ciencias Sociales, UBA. Recuperado de http:// bibliotecavirtual.clacso.org.ar/Argentina/iigg-uba/20100720094530/dt48.pdf

Schuster, F. L., \& Scribano, A. (2001). Protesta social en la Argentina de 2001: entre la normalidad y la ruptura. OSAL (Observatorio Social de América Latina), (5), 17-22.

Svampa, M. (2009). Protesta, Movimientos Sociales y Dimensiones de la acción colectiva en América Latina. Madrid: Universidad Complutense de Madrid - Fundación Carolina. Recuperado de http://maristellasvampa.net/archivos/ensayo57.pdf

Svampa, M. (2018). Movimientos sociales, tradiciones políticas y dimensiones de la acción colectiva en América Latina. En S. Gómez Obando, C. Moore Torres, y L. Múnera Ruiz (Eds.), Los sa- 
beres múltiples y las ciencias sociales y políticas (pp. 31-63). Bogotá: Universidad Nacional de Colombia. Facultad de Derecho, Ciencias Políticas y Sociales. Instituto Unidad de Investigaciones Jurídico Sociales Gerardo Molina (UNIJUS)
Tarrow, S. (1997). El poder en movimiento: los movimientos sociales, la acción colectiva y la política. Madrid: Alianza Editorial.

Zibechi, R. (2017). Movimientos sociales en América Latina: $\mathrm{El}$ "mundo otro" en movimiento. Bogotá D.C.: Ediciones Desde Abajo. 